\title{
A "long-standing" malpositioned pacing lead. Long-term follow-up after extraction
}

\author{
Berardo Sarubbi1, Giancarlo Scognamiglio', Flavia Fusco', Enrico Melillo1, Michele D'Alto1, \\ Maria Giovanna Russo²
}

\author{
1 Adult Congenital Heart Disease Unit, Monaldi Hospital, Naples \\ 2 Department of Paediatric Cardiology, Second University of Naples, Monaldi Hospital, Naples, Italy
}

\begin{abstract}
Transvenous pacemaker (PM) catheters can be unintentionally placed in the left ventricle (LV) during the implantation procedure. An 8 -year-old girl was discovered with a malpositioned pm wire, seven years after the implant. Trans-thoracic echocardiogram revealed the lead traversing the inter-atrial septum, crossing the mitral valve and embedded in the basal lateral wall of the LV. This is a report of a 14-year long follow-up after the surgical extraction of the malpositioned PM lead.
\end{abstract}

\section{Introduction}

Transvenous pacing can be certainly performed in infants and small children, and it has been demonstrated in the neonate as well [1-2]. As pacemaker (PM) generators become smaller and lead diameters become thinner, placing a permanent transvenous pacing system has be-

Corresponding author: Dr. Berardo Sarubbi, Adult Congenital Heart Disease Unit, Monaldi Hospital, Via Leonardo Bianchi, 80131 Naples, Italy. Tel. +39.081.7065288. E-mail: berardo.sarubbi@virgilio.it

Key words: Congenital AV block; pacemaker malposition; surgical extraction.

Contributions: BS, manuscript writing and case supervision; GS, FF, manuscript writing and data collection; EM, MDA, manuscript writing, electrophysiological study performance; MGR, case supervision.

Conflict of interest: The authors declare no conflict of interest.

Acknowledgments: The authors would like to thank all the medical and nursing staff for taking care of the patient.

Received for publication: 19 March 2018

Accepted for publication: 11 July 2018

(C) Copyright B. Sarubbi et al., 2018

Tipografia PI-ME Editrice, Italy

Monaldi Archives for Chest Disease 2018; 88:927

doi: 10.4081/monaldi.2018.927

This article is distributed under the terms of the Creative Commons Attribution Noncommercial License (by-nc 4.0) which permits any noncommercial use, distribution, and reproduction in any medium, provided the original author(s) and source are credited. come easier. Although nowadays a transvenous lead can be placed in children, there is a greater risk of complication in small patients [3]. One extremely rare complication associated with transvenous PM implantation is the inadvertent positioning of pacing lead in the left ventricle [4]. The greater risks depend on the delay of diagnosis of lead malpositions. This report concerns a case of long

standing malpositioned PM wire in a paediatric patient and the successive long term follow up after the successful surgical extraction.

\section{Case report}

A one-year-old child underwent an endocardial PM implantation for a congenital atrioventricular (AV) block in a different Hospital. Seven years later, she was referred to our cardiac tertiary centre because of a clinical assessment for the presence of a cardiac murmur, never heard before by the paediatrician.

Physical examination revealed an average build young girl who was in no apparent distress state and had stable vital signs: regular pulse of $75 \mathrm{bpm}$ and blood pressure of 110/60 mmHg. Cardiac examination was significant for a $2 / 6$ systolic murmur at apex with irradiation to the axilla. The ECG revealed a ventricular PM rhythm with a right bundlebranch block (RBBB) morphology (Figure 1A). Chest radiography identified a lead directed posteriorly, giving the suspicion of an abnormal location of the ventricular pacer wire (Figure 1 B-C). Trans-thoracic echocardiogram revealed normal LV function, no pericardial effusion, and an abnormal bright linear structure consistent with a PM lead passing through a patent foramen oval and crossing the mitral valve to embed in the basal lateral wall of the $\mathrm{LV}$, causing a mild to moderate mitral regurgitation (Figure 1D).

After informed consent was obtained, in accordance with the guidelines of our institution, an electrophysiological (EP) study was performed. Although complete EP evaluation was performed, this report deals only with the results of conduction system assessment. The EP study was performed after mild sedation with two $5 \mathrm{~F}$ quadripolar catheters positioned in the high right atrium and His bundle region. The SA, AH, HV and VV intervals, as well as the conduction system refractory periods, were defined and measured as conventionally described. Switching-off the PM, the following data were obtained:

- Normal sinus node function.

- Third-degree AV block with a junctional "escape" rhythm of 58 bpm (QRS duration:70 ms).

- A normal infra-His conduction time (HV=39 ms) (Figure 2).

- After intravenous injection of $1 \mathrm{mg}$ of atropine we revealed persistent third-degree AV block with a faster escape rhythm (the HR increase was more than the $50 \%$ of the initial HR).

The patient was offered the surgical extraction of the PM wire via thoracotomy and possible mitral valve repair. 
After appropriate evaluation, the small patient underwent the surgical extraction of the PM lead, and, in the same surgical session, plastic of the mitral valve, closure of the atrial septal defect with a Dacron patch and removal of PM generator. Since then she has been on ACE-I therapy and she has been followed at our outpatient clinic in order to undergo ECG, echocardiography, 24H ECG Holter and ECG stress test every 6 to 12 months for 14 years. She never suffered from syncope or presyncope. The ECG-Holters never recorded significant pauses. The patient is now 22 years-old, she has a good quality of life and she is in I-II NYHA class. At the last outpatient visit, the ECG showed AV dissociation for third-degree BAV with tight QRS junctional escape rhythm (QRS $90 \mathrm{~ms}$ ) at the rate of $53 \mathrm{bpm}$ (Figure 3). At the last cardiological assessment, the ecocardiography found in situ interatrial patch without any residual shunts, mild to minimal mitralic regurgita- tion, mild dilatation of the left atrium, left ventricular with spherical shape but with preserved global pump function (EF:65\%). The treadmill ECG stress test was stopped at the III stadium of Bruce protocol because of muscle fatigue, with evidence of an adequate exertional increase in heart rate (from $61 \mathrm{bpm}$ in basal condition to $123 \mathrm{bpm}$ that is $61 \%$ of theoretical max HR).

\section{Discussion}

Left ventricular location of a PM lead, although rare, is a wellknown complication after PM implantation. The clinical suspicion is based on the results of physical examination and simple tests such as ECG and chest radiography. Twelve-lead ECG is a rapid and reli-
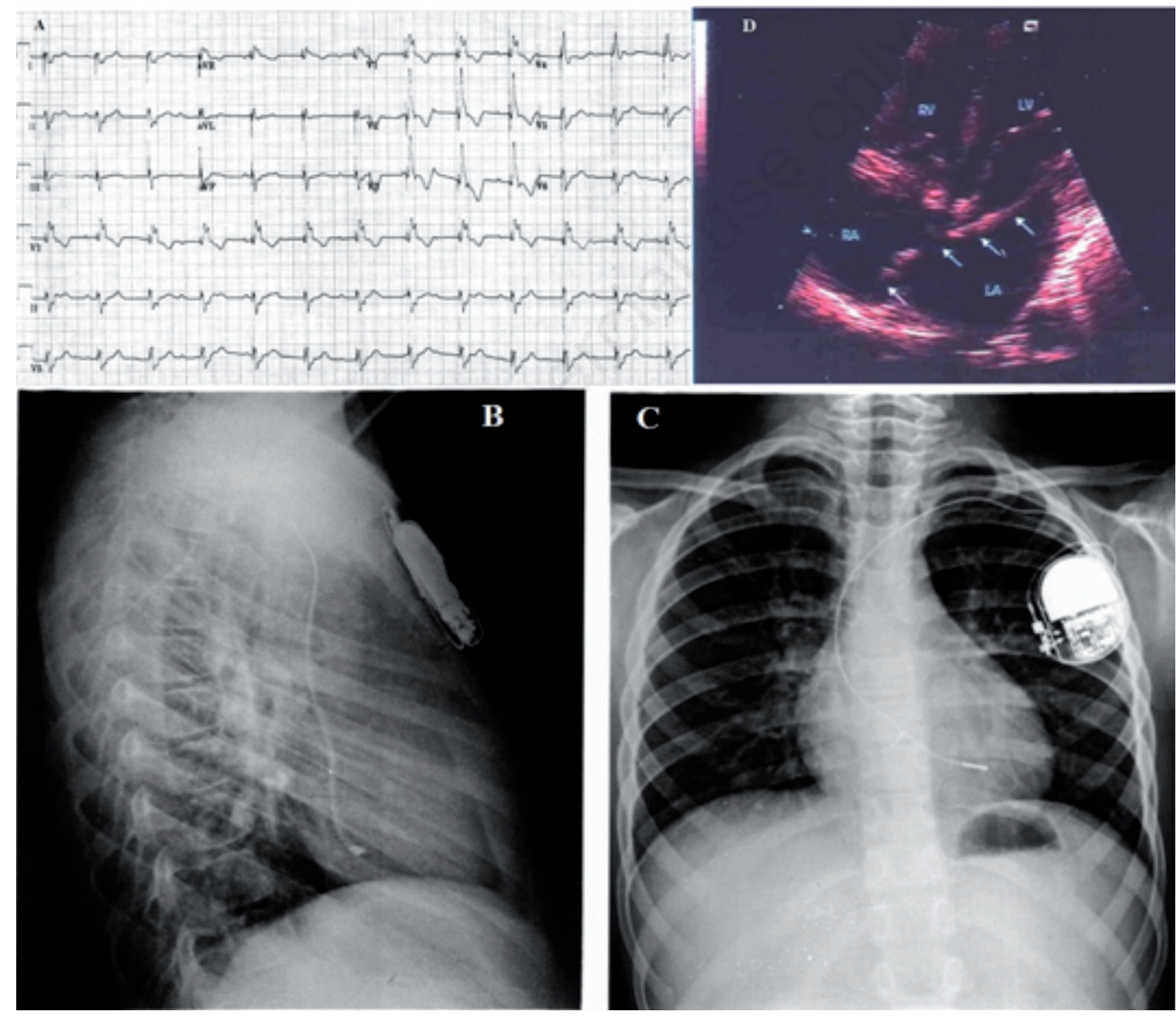

Figure 1. A) Twelve-lead standard ECG at the admission (aged 8 years). Note the ventricular pacemaker rhythm with a right bundle-branch block (RBBB) morphology. B) LL chest radiography. Note the pacemaker wire directed posteriorly. C) AP chest radiography. Note the pacemaker wire directed posteriorly. D) Trans-thoracic echocardiogram. Note the lead traversing the interatrial septum crossing the mitral valve and embedding in the basal lateral wall of the left ventricle. 
able instrument to recognize right and left ventricular pacing morphologies. The expected morphology of right ventricular pacing is a left bundle branch block (LBBB) pattern. Early activation of the left ventricle by the malpositioned lead creates a RBBB pattern which is a very helpful clue in the diagnosis [5]. However, a RBBB pattern during ventricular pacing may also occur if the lead is positioned in the coronary sinus, or if it perforates the right ventricle or the interventricular septum, or in case of left ventricle epicardial pacing or even with a normal placed lead in the right ventricle [4-5]. So, the reliance on intraoperative monitors for detection of RBBB may be misleading as this pattern may not be displayed by the recording channels [6]. Moreover, on chest X-ray a postero-anterior view is not sufficient to identify the position of a ventricular pacing lead; a lateral view is essential to demonstrate an abnormal posterior position of the catheter tip, as opposed to the anterior position expected with a right ventricular placed lead. Indeed, a posterior catheter position may also be due to an electrode in the coronary sinus or in the interventricular vein [4-5]. Thus, although ECG and chest X-ray should raise the suspicion, neither of them can diagnose left ventricular catheter placement with complete certainty. Transthoracic or transoesophageal echocardiography are usually the tests of choice to confirm the exact lead position [7].
If X-ray or transthoracic/transoesophageal echocardiography are both insufficient to confirm the exact lead position, in presence of not clear results contrast-enhanced computed tomography scan (CTA) of the heart and great vessels can sometimes provide the exact information of PM lead location.

Misplacement of a PM catheter intended for the right ventricle can be a result of the catheter traversing a patent foramen ovale or an atrial septal defect, of the interatrial or interventricular septum perforation by the catheter, or of direct lead placement in left ventricle through the brachial, axillary or subclavian artery, which could be mistaken for a vein $[4,8,9]$.

In our patient, the most likely scenario was a lack of recognition of the route of the PM lead during implantation by fluoroscopy. Probably, during the procedure, the bend of the PM lead was misinterpreted as the lead traversing the tricuspid annulus when indeed it was going through the PFO. In addition, despite the long-term follow-up, the abnormal location of the lead was not recognized on chest radiography or on ECG.

For this reason, it should be emphasised that in each case presenting with newly detected RBBB paced QRS morphology, physicians should seek immediate echocardiography to precisely assess the position of the ventricular lead. The possibility of the initial normal po-

Divisione di Cardiologia Pediatrica - II Universita' di Napoli - A.O. Monaldi

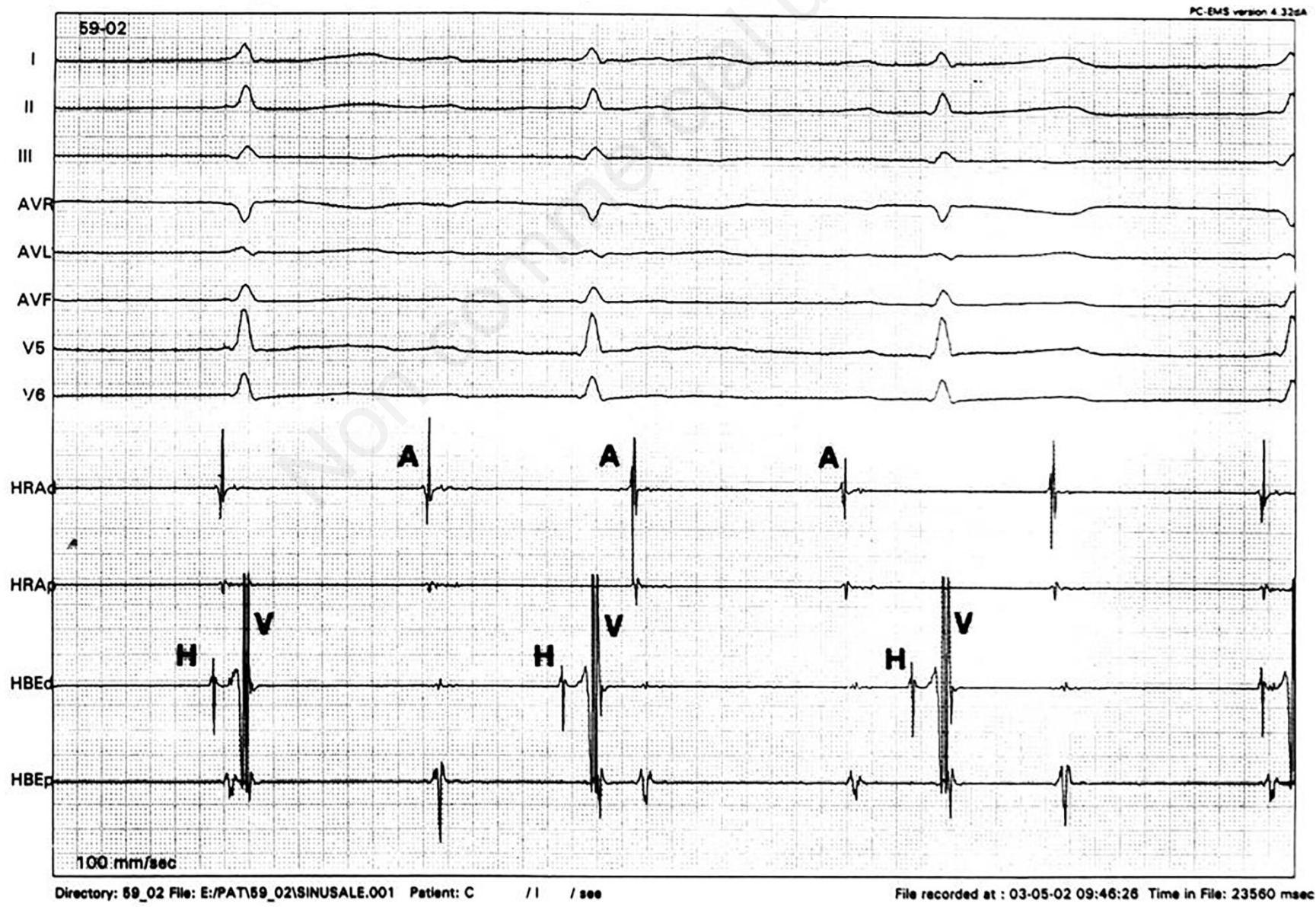

Figure 2. Electrophysiologic study, switching-off the pacemaker: third degree AV block with a junctional "escape" rhythm (QRS duration: $70 \mathrm{~ms}$ ) of $58 \mathrm{bpm}$ and normal infra-His conduction time (HV=39 ms). HRAd, high right atrium-distal electrodes; HRAp, high right atriumproximal electrodes HBEd, His bundle electrogram-distal electrodes; HBEp, His bundle electrogram-proximal electrodes). 
sitioning in the right ventricle with the following dislodgement through the PFO into the left ventricle is an extremely unlikely scenario. A malposition of a PM catheter in the left ventricle can be asymptomatic or can lead to significant morbidity from thrombi and following debilitating cerebral stroke $[4,10,11]$. However, the greater risks are usually depending on failure of timely diagnosis once lead malposition occurs. A PM malpositioned catheter in the left ventricle should be removed.

In our case, at the time of malpositioned wire discovery, a long-life anticoagulation therapy was considered an alternative approach to the extraction [4]. Yet, this approach was not strongly suggested to the patient's parents because of the possible risks due to chronic anticoagulation in terms of haemorrhages but also because we considered the possible damages to mitral valve apparatus as a consistent complication of the lead malposition and we thought the permanence of the lead might increase mitral regurgitation over time.

Transvenous pacemaker catheter extraction from the right ventricle has become a common procedure. However, with catheters in the left ventricle there may be a risk of dislodging or stripping off thrombotic material during attempted percutaneous extraction, especially if a catheter must be withdrawn through a PFO. However, in our case, also the effective necessity of a permanent PM should be discussed. We are unaware of the patient's clinical status at the PM implantation time. The presence at that time of low cardiac output together with third-de- gree AV block cannot be excluded. The possibility of a ventricular rate lesser than 50 to $55 \mathrm{bpm}$, other condition for which there is a class I evidence that the permanent PM is useful [9], is an unlikely scenario as the patient showed a higher spontaneous heart rate when she was 8 years of age.

We have to consider that the results of the EP study strongly influenced the decision of extraction without pacemaker re-implantation.

During our 14 years follow up post-extraction the patient did not develop ventricular dysfunction and at our tests she never showed chronotropic incompetence limiting the physical activity, long pauses, wide QRS complexes, prolonged QT interval, mean ventricular rate $<50$ bpm or complex ventricular ectopy which could justify the implantation of a PM in an asymptomatic patient with congenital AV block [9].

\section{Conclusions}

This case is an example of a probable useless and damaging PM implantation in a very young girl who had an extremely rare complication, that is the malposition of the pacing lead in the left ventricle. The wire has remained in a wrong position for seven years before the mistake was acknowledged. Luckily, the patient had no embolic events and she had a favourable course of the follow-up after the surgical extraction.
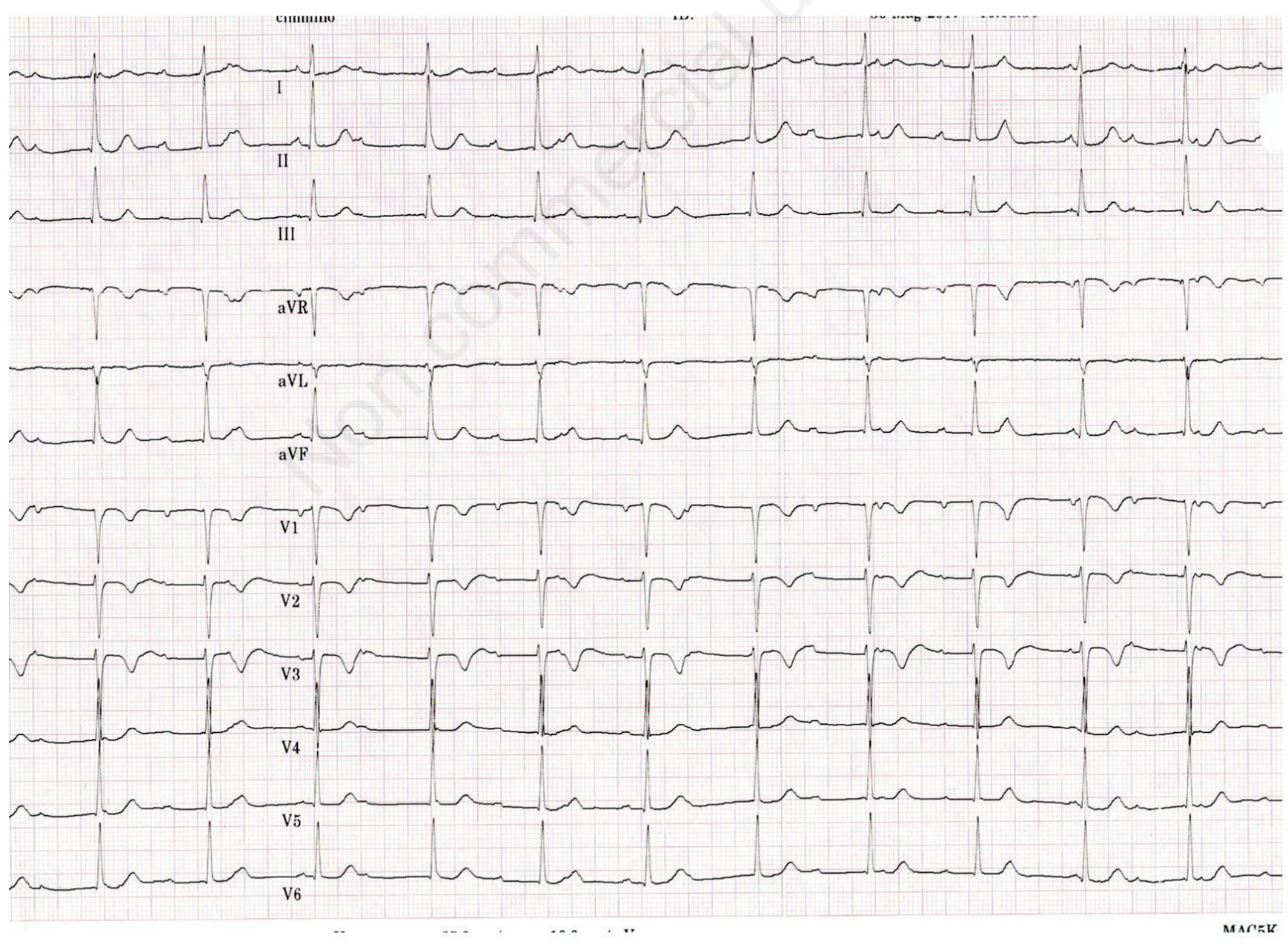

Figure 3. Twelve-lead standard ECG at the last cardiological routine admission (aged 22 years). 


\section{References}

1. Konta L, Chubb M.H, Bostock J, et al. Twenty-seven years experience with transvenous pacemaker implantation in children weighing <10 kg. Circ Arrhythm Electrophysiol 2016;9:e003422.

2. Vos LM, Kammeraad JAE, Freund MW, et al. Long-term outcome of transvenous pacemaker implantation in infants: a retrospective cohort study. Europace 2017;19:581-7.

3. Van Gelder BM, Bracke FA, Oto A, et al. Diagnosis and management of inadvertently placed pacing and ICD leads in the left ventricle: a multicenter experience and review of the literature. Pacing Clin Electrophysiol 2000;23:877-83.

4. Gondi B, Nanda NC. Real-time, two-dimensional echocardiographic features of pacemaker perforation. Circulation 1981;64:97-106.

5. Tzeis S, Andrikopoulos G, Weigand S, et al. Right bundle branch block-like pattern during uncomplicated right ventricular pacing and the effect of pacing site. Am J Cardiol 2016;117:935-59.

6. Vanhercke D, Heytens W, Verloove H. Eight years of left ventricle pacing due to inadvertent malposition of a transvenous pacemaker lead in the left ventricle. Eur $\mathrm{J}$ Echocardiogr 2008;9:825-7.

7. Arnar D0, Kerber RE. Cerebral embolism resulting from a transvenous pacemaker catheter inadvertently placed in the left ventricle: a report of two cases confirmed by echocardiography. Echocardiography 2001; 18:681-684.

8. Boczar K, Komar M, Ząbek A, et al. Spontaneous dislocation of the endocardial lead into the left ventricle through the intraventricular septum. Kardiol Pol 2017;75:79.

9. Ząbek A, Małecka B, Pfitzner R, et al. Extraction of left ventricular pacing lead inserted via the left subclavian artery. Pol Arch Med Wewn 2013;123:560-1.

10. Aslam AA, Mcllwain EF, Talano JV, et al. An unusual case of embolic stroke: a permanent ventricular pacemaker lead entirely within the arterial system documented by transthoracic and transesophageal echocardiography. Echocardiography 1999;16:373-8.

11. Brignole M, Auricchio A, Baron-Esquivias G, et al. 2013 ESC Guidelines on cardiac pacing and cardiac resynchronization therapy. The Task Force on cardiac pacing and resynchronization therapy of the European Society of Cardiology (ESC). Developed in collaboration with the European Heart Rhythm Association (EHRA). Eur Heart J 2013;34:2281-329. 